\title{
Conserved Molecular Mechanism of TyrA Dehydrogenase Substrate Specificity Underlying Alternative Tyrosine Biosynthetic Pathways in Plants and Microbes
}

\author{
Craig A. Schenck ${ }^{\dagger}$, Yusen Men and Hiroshi A. Maeda* \\ Department of Botany, University of Wisconsin-Madison, Madison, WI, United States
}

OPEN ACCESS

Edited by:

Robert Stephen Phillips,

University of Georgia, United States

Reviewed by:

David Douglas Boehr,

Pennsylvania State University,

United States

Michael D. Toney,

University of California, Davis,

United States

*Correspondence:

Hiroshi A. Maeda

maeda2@wisc.edu

${ }^{\dagger}$ Present Address:

Craig A. Schenck,

Department of Biochemistry and Molecular Biology, Michigan State

University, East Lansing, MI,

United States

Specialty section:

This article was submitted to

Structural Biology,

a section of the journal

Frontiers in Molecular Biosciences

Received: 09 August 2017

Accepted: 24 October 2017

Published: 07 November 2017

Citation:

Schenck CA, Men Y and Maeda HA

(2017) Conserved Molecular

Mechanism of TyrA Dehydrogenase

Substrate Specificity Underlying

Alternative Tyrosine Biosynthetic

Pathways in Plants and Microbes.

Front. Mol. Biosci. 4:73.

doi: 10.3389/fmolb.2017.00073
L-Tyrosine (Tyr) is an aromatic amino acid synthesized de novo in plants and microbes. In animals, Tyr must be obtained through their diet or synthesized from L-phenylalanine. In addition to protein synthesis, Tyr serves as the precursor of neurotransmitters (e.g., dopamine and epinephrine) in animals and of numerous plant natural products, which serve essential functions in both plants and humans (e.g., vitamin E and morphine). Tyr is synthesized via two alternative routes mediated by a TyrA family enzyme, prephenate, or arogenate dehydrogenase $\left(\mathrm{PDH} / \mathrm{Tyr} \mathrm{A}_{\mathrm{p}}\right.$ or $\left.\mathrm{ADH} / \mathrm{Ty} \mathrm{r} \mathrm{A}_{a}\right)$, typically found in microbes and plants, respectively. Although $\mathrm{ADH}$ activity is also found in some bacteria, the origin of arogenate-specific TyrA $\mathrm{A}_{a}$ enzymes is unknown. We recently identified an acidic Asp222 residue that confers ADH activity in plant TyrAs. In this study, structure-guided phylogenetic analyses identified bacterial homologs, closely-related to plant TyrAs, that also have an acidic 222 residue and ADH activity. A more distant archaeon TyrA that preferred PDH activity had a non-acidic Gln, whose substitution to Glu introduced ADH activity. These results indicate that the conserved molecular mechanism operated during the evolution of arogenate-specific Tyr $\mathrm{A}_{a}$ in both plants and microbes.

Keywords: L-Tyrosine, primary metabolism, natural products, aromatic amino acid, substrate specificity

\section{INTRODUCTION}

L-Tyrosine (Tyr) is an aromatic amino acid required for protein synthesis in all organisms, but synthesized de novo in plants and microbes. Thus, in animals Tyr must be acquired through the diet or produced from L-phenylalanine (Phe) by Phe-hydroxylase (Fitzpatrick, 1999). In addition to protein synthesis, Tyr is used to synthesize animal neurotransmitters, such as dopamine and epinephrine (adrenaline) (Fernstrom and Fernstrom, 2007) and melanin skin pigments (Slominski et al., 2004). Tyr also serves as the precursor to numerous plant natural products with diverse functions such as electron carriers (e.g., plastoquinone and ubiquinone; Millner and Barber, 1984), defense (e.g., dhurrin and rosmarinic acid; Petersen, 2013; Gleadow and Møller, 2014), and pollinator attraction (e.g., betalain pigments; Gandía-Herrero and García-Carmona, 2013). Some of these natural products also serve medicinal and nutritional roles in humans such as antioxidants (vitamin E; Falk and Munné-Bosch, 2010), and analgesics (e.g., morphine; Sato et al., 2007).

Tyr is synthesized from prephenate, downstream of the shikimate pathway, by two alternative routes. In most microbes prephenate is first converted into 4-hydroxyphenylpyruvate 


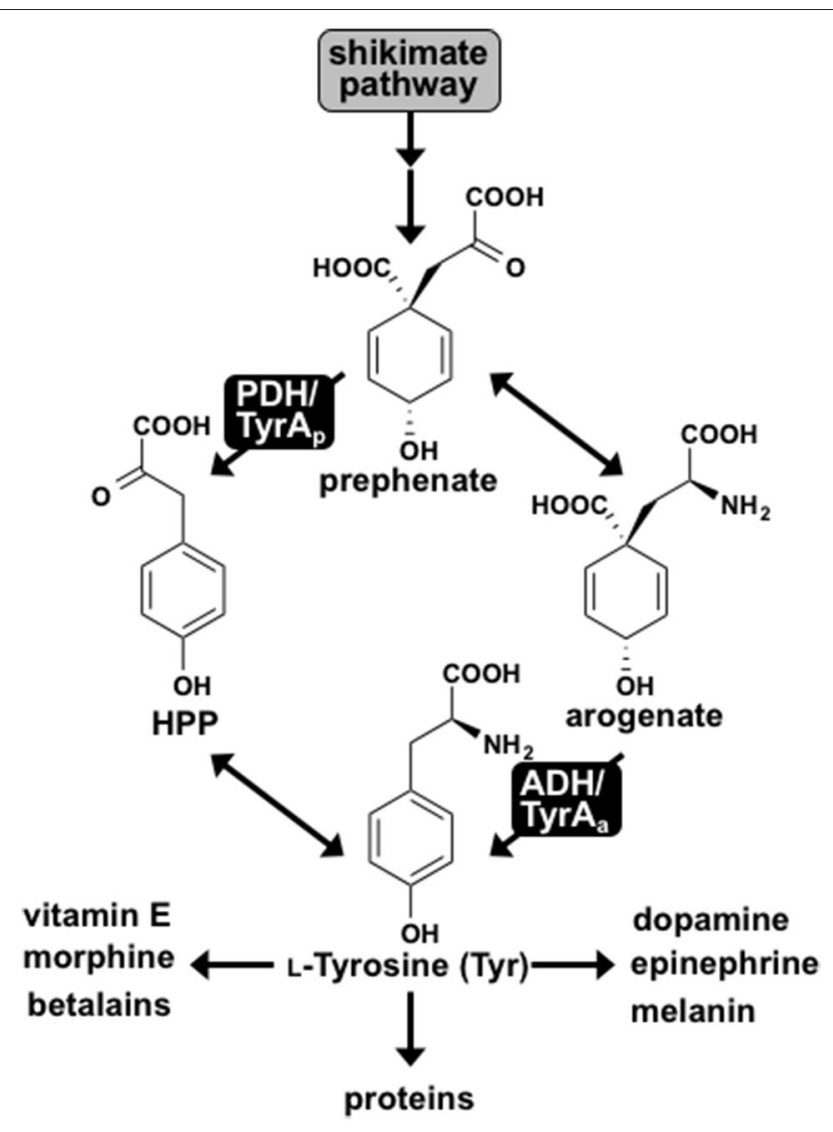

FIGURE 1 | L-Tyrosine biosynthesis via two pathways in plants and microbes. Two pathways for Tyr biosynthesis from prephenate downstream of the shikimate pathway are shown. The $\mathrm{PDH} / \mathrm{Ty}_{\mathrm{A}} \mathrm{p}$ pathway is present in most microbes, whereas the ADH/TyrA $\mathrm{A}_{a}$ pathway is ubiquitous in plants. Tyr serves as the precursor for protein synthesis and many downstream metabolites in plants and humans.

(HPP) by a $\mathrm{NAD}^{+}$-dependent prephenate-specific TyrA dehydrogenase $\left(\mathrm{PDH} / \mathrm{TyrA}_{\mathrm{p}}\right)$, followed by transamination to form Tyr (Figure 1) (Jensen and Pierson, 1975; Bentley, 1990). In contrast, plants first transaminate prephenate to form arogenate, which is converted to Tyr by a $\mathrm{NADP}^{+}$-dependent arogenatespecific TyrA dehydrogenase ( $\mathrm{ADH} / \mathrm{TyrA}_{\mathrm{a}}$ ) (Gaines et al., 1982; Connelly and Conn, 1986; Rippert and Matringe, 2002). Plant $\operatorname{TyrA}_{\mathrm{a}}$ and microbial TyrA $\mathrm{p}$ catalyze the key regulatory step in Tyr biosynthesis, and their substrate specificity defines the Tyr biosynthetic routes via arogenate and prephenate intermediate, respectively (Figure 1).

Notably, exceptions have been reported for the typical cofactor and substrate specificities of TyrA dehydrogenases in plants and microbes. Some microbes, for example, use $\mathrm{NADP}^{+}$cofactor instead of $\mathrm{NAD}^{+}$(Fazel et al., 1980; Subramaniam et al., 1994). Arogenate-specific TyrA $\mathrm{a}_{\mathrm{a}}$ enzymes have also been identified in some microbes, such as the $\alpha$-proteobacteria Zymomonas mobilis and Phenylobacterium immobile (Mayer et al., 1985; Zhao et al., 1993). While all plants investigated have arogenate-specific TyrA $_{a}$, the legume family additionally possesses prephenatespecific TyrA $A_{p}$ enzymes (Gamborg and Keeley, 1966; Rubin and
Jensen, 1979; Schenck et al., 2015). Using the unique presence of TyrA $A_{p}$ in legumes combined with structural analyses, a single acidic residue in the active site, Asp222, was recently shown to confer arogenate substrate specificity of plant TyrAs by directly interacting with the side chain amine of arogenate substrate (Schenck et al., 2017), which is absent in prephenate (Figure 1). Indeed, mutating Asp222 of diverse plant $\mathrm{TyrA}_{\mathrm{a}}$ into the corresponding Asn residue in legume TyrA $\mathrm{A}_{\mathrm{p}}$ reduced their $\mathrm{ADH}$ activity and introduced $\mathrm{PDH}$ activity, suggesting that Asn222 played a key role in the recent evolution of legume-specific $\mathrm{TyrA}_{\mathrm{p}}$ (Schenck et al., 2017). However, the early evolutionary origin and mechanism of microbial and plant $\mathrm{TyrA}_{\mathrm{a}}$ enzymes are still unresolved.

Here, we used the Asp222 residue to trace the evolutionary history of $\mathrm{TyrA}_{\mathrm{a}}$ enzymes in deep taxonomic lineages across Plantae and microbes. Structure-guided, phylogenetic analyses combined with biochemical characterization show that microbial TyrA orthologs closely-related to plant TyrA also have a corresponding Asp (or Glu) residue and prefer $\mathrm{ADH}$ activity. Contrarily, microbial TyrA, which are more distantly-related to plant TyrAs, contained a non-acidic Gln or Asn and preferred PDH activity. Furthermore, site-directed mutagenesis of an acidic Asp into a neutral Asn on a spirochaetes $\operatorname{TyrA}_{a}$ reduced $\mathrm{ADH}$ activity, while introducing $\mathrm{PDH}$ activity. The reciprocal mutation of Gln into an acidic Glu on an archaeon TyrA $_{p}$ reduced $\mathrm{PDH}$ and introduced $\mathrm{ADH}$ activity. These data suggest that plants and some microbial TyrA orthologs share an evolutionarily conserved substrate specificity mechanism, and that acquisition of the key active site acidic residue was crucial in evolution of arogenate-specific TyrA $\mathrm{A}_{\mathrm{a}}$ enzymes in plants and closely-related microbes.

\section{MATERIALS AND METHODS}

\section{Identification of Microbial TyrA Orthologs}

BlastP searches were performed using the amino acid sequences of previously characterized TyrA homologs from plants [soybean PDH; GmPDH1 (Schenck et al., 2015) and Arabidopsis ADH; AtADH2 (Rippert and Matringe, 2002)] and microbes [Synechocystis sp. PCC6803 ADH (Legrand et al., 2006), and E. coli PDH (Hudson et al., 1984)] as the query in the NCBI database. This yielded only closely-related plant and microbial TyrA orthologs (e.g., algae and, $\gamma$-proteobacteria), which were then used as the query to perform additional BlastP searches. Every 5th BlastP hit was selected to provide sequences from various microbial lineages and limit bias in sample selection. Data S1 contains all the sequence information for the TyrA orthologs used in Figure 2 and Figure S1. A structure-guided amino acid alignment was performed in PROMALS3D (Pei and Grishin, 2007) using the default parameters with structures of TyrA enzymes from plants and microbes with varying substrate specificities (G. max TyrA ; GmPDH1; PDB \# 5T8X, $H$. influenzae TyrA $;$; HiPDH; 2PV7, and Synechocystis sp. PCC6803 TyrA ; SynADH; PDB \# 2F1K). The amino acid alignment from PROMALS3D was used to construct phylogenetic trees using MEGA7 (Kumar et al., 2016). The full amino acid alignment can be found in Data S2. The analyses involved 130 amino acid 

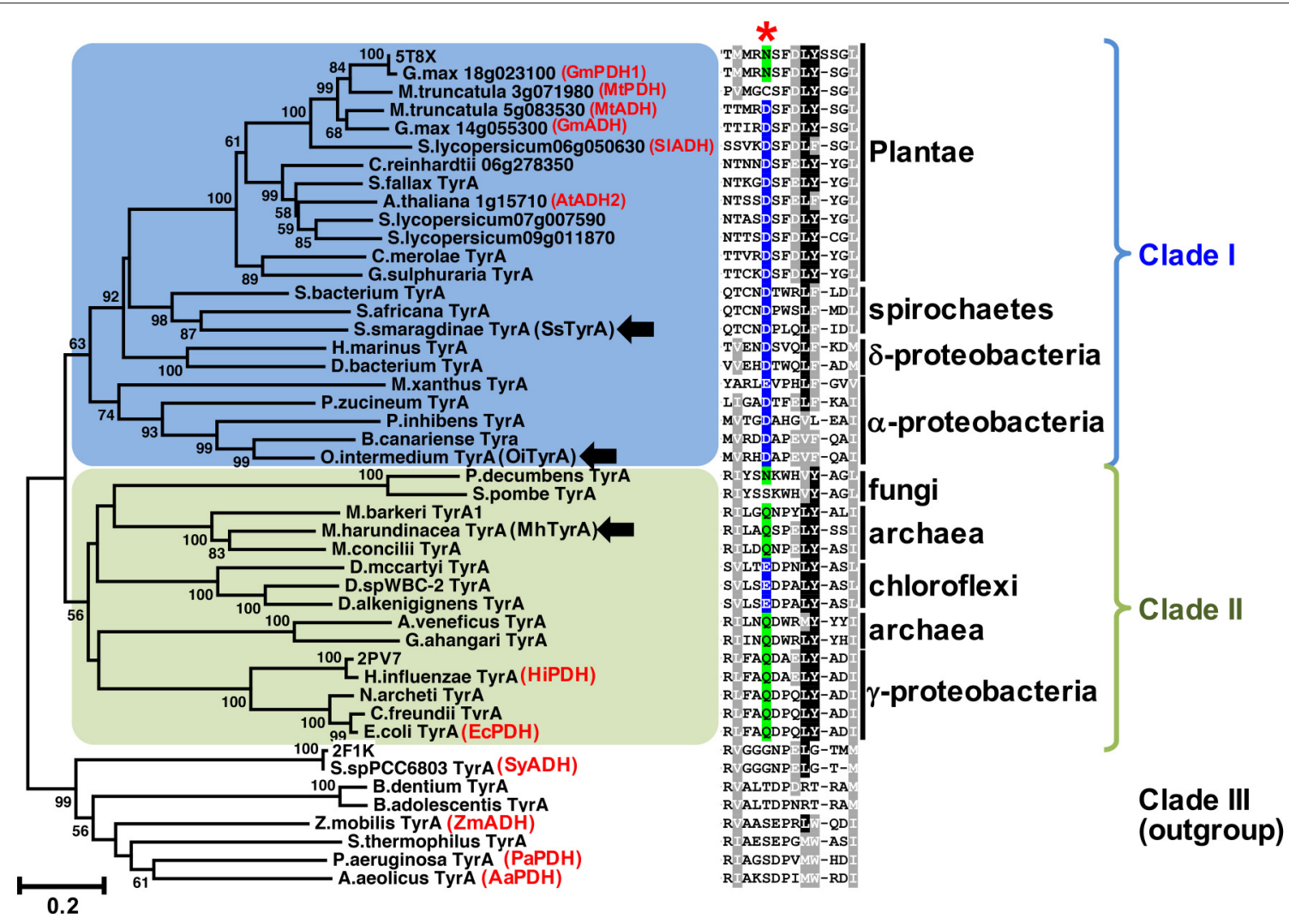

FIGURE 2 | The conserved acidic residue at 222 among clade I TyrA orthologs from plants, algae, and closely-related bacteria. Structure-guided phylogenetic analysis of plant and microbial TyrAs. Three distinct clades are formed; clade I contains all plant TyrAs and closely-related microbes (blue), clade II contains bacteria, archaea, and fungi TyrAs (green), and clade III, which was used as an outgroup. Enzymes characterized in this study are marked by black arrows. Structures used to guide the alignment are labeled with their PDB IDs. Previously characterized TyrAs are labeled in red with their preferred PDH or ADH activity. Scale bar represents number of substitutions per branch length. A trimmed amino acid alignment of corresponding sequences shows a conserved acidic residue (Asp or Glu, highlighted in blue) among clade I, which is replaced with a non-acidic Asn or Gln residue (highlighted in green) in most clade II at the corresponding 222 position marked with a red star. Identical amino acids present in $>50 \%$, black shading; biochemically similar residues present in $>50 \%$ of the sequences, gray shading.

sequences and all sites with $<75 \%$ coverage were eliminated from the analysis. A neighbor-joining method (Figure S1A; Saitou and Nei, 1987) was used to estimate evolutionary history using 1,000 bootstrap replicates (values shown at branches). The tree in Figure 2 is a representative tree using a subset of the sequences found in Figure S1. Additional phylogenetic analyses were performed using the Maximum Likelihood method based on the Jones-Taylor-Thornton (JTT) matrix-based model (Jones et al., 1992), which gave overall similar results (Figure S1B). All phylogenetic trees are drawn to scale, with branch lengths measured in the number of substitutions per site.

\section{Recombinant Protein Expression and Purification and Site Directed Mutagenesis}

Full length coding sequences from Ochrobactrum intermedium LMG 3301 (EEQ93947.1; OiTyrA), Sediminispirochaeta smaragdinae DSM 11293 (ADK80640.1; SsTyrA), and Methanosaeta harundinacea (KUK94425.1; MhTyrA) were codon optimized for expression in E. coli, gene synthesized (Biomatik), and inserted into pET28a vector using EcoR1 and
Nde1 sites in frame with an N-terminal $6 \mathrm{x}$-His tag using a previously described cloning method (Schenck et al., 2015).

For site directed mutagenesis, plasmid template was diluted 100-fold, mixed with $0.04 \mathrm{U} / \mu \mathrm{L}$ Phusion DNA polymerase (Thermo), $0.2 \mathrm{mM}$ dNTP's, $0.5 \mu \mathrm{M}$ forward and reverse mutagenesis primers and $1 \mathrm{x}$ Phusion reaction buffer (Thermo), and then placed in a thermocycler for $98^{\circ} \mathrm{C}$ for $30 \mathrm{~s}$ followed by 20 cycles of $10 \mathrm{~s}$ at $98^{\circ} \mathrm{C}, 20 \mathrm{~s}$ at $70^{\circ} \mathrm{C}$, $4.5 \mathrm{~min}$ at $72^{\circ} \mathrm{C}$ with a final extension at $72^{\circ} \mathrm{C}$ for $10 \mathrm{~min}$. The sequence of primers used for mutagenesis were $\left(5^{\prime}\right.$ CATTCTGGCCGAAAGCCCGGAACTGTATAGTAGC-3') and $\left(5^{\prime}\right.$-GTTCCGGGCTTTCGGCCAGAATGCGGCCCACA AAATC- $\left.3^{\prime}\right)$ for MhTyrA and (5'-GTAACAATCCACTTCAGCT GTTTATAGACTTGCAAC- $\left.3^{\prime}\right)$ and (5'-CTGAAGTGGATTG TTACACGTTTGTTCGCGCACCTG-3') for SsTyrA (mutated codons are italicized). The PCR products were purified with QIAquick Gel Extraction Kit (Qiagen), treated with DpnI (Thermo) to digest methylated template DNA for $30 \mathrm{~min}$ at $37^{\circ} \mathrm{C}$, and then transformed into E. coli XL1-Blue cells. Plasmids were sequenced to confirm that no errors were introduced during PCR and cloning. 

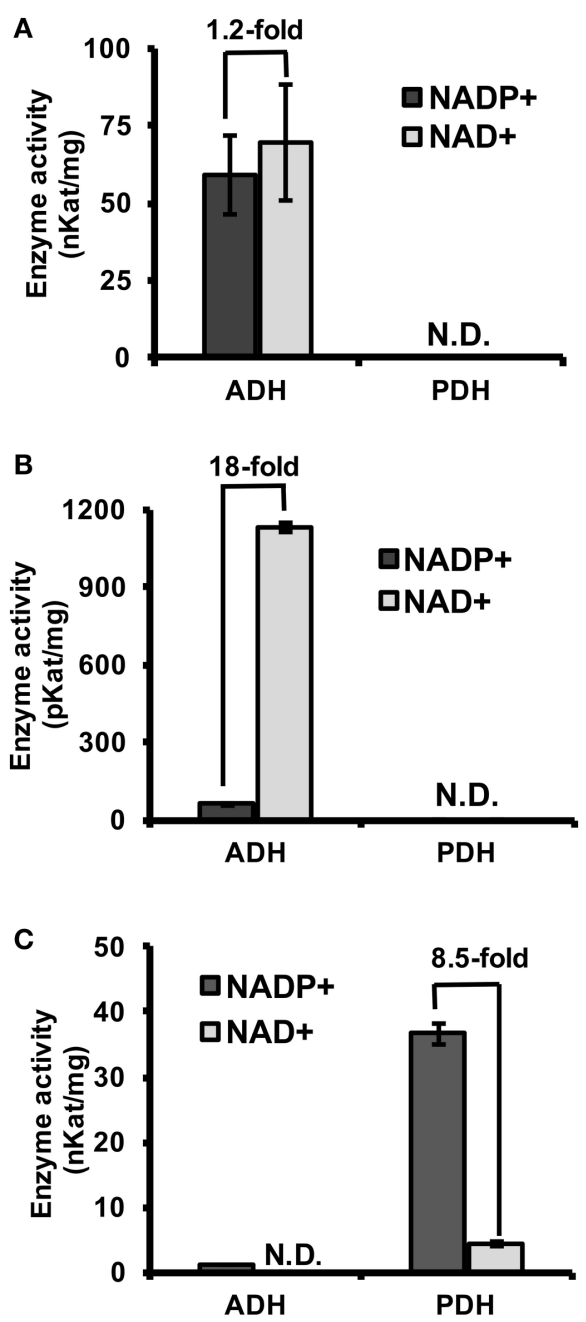

FIGURE 3 | Substrate and cofactor specificity of microbial TyrA orthologs. $\mathrm{ADH}$ and $\mathrm{PDH}$ assays were performed with $0.8 \mathrm{mM}$ arogenate and prephenate, respectively, and $0.8 \mathrm{mM}$ cofactor (NADP ${ }^{+}$, black; $\mathrm{NAD}^{+}$, gray). (A) Purified recombinant SsTyrA (spirochaetes) was used to test enzymatic activity, and shown as the average in $\mathrm{nKat} / \mathrm{mg}$ protein \pm SEM of $n=3$. (B) $\alpha$-proteobacteria TyrA (OiTyrA) cell lysate was used as purification of the recombinant enzyme was not successful. Average enzymatic activity is shown as $\mathrm{pKat} / \mathrm{mg}$ protein \pm SEM of $n=3$ (C) Purified recombinant MhTyrA (archaea) was used to test enzymatic activity, and shown as the average in $\mathrm{nKat} / \mathrm{mg}$ protein \pm SEM of $n=3$. N.D. no activity detected. Cofactor preference is indicated by the fold-change over the bars.

For recombinant protein expression, E. coli Rosetta2 (DE3) cells (Novagen) transformed with the above plasmids were cultured as previously reported (Schenck et al., 2017). For protein purification, $20 \mathrm{~mL}$ of the E. coli supernatant expressing the appropriate plasmid was applied to a $1 \mathrm{~mL}$ HisTrap FF column for purification of the His-tagged recombinant protein using an ÄKTA FPLC system (GE Healthcare). After loading the supernatant, the column was washed with 20 column volumes of $90 \%$ buffer $\mathrm{A}(0.5 \mathrm{M} \mathrm{NaCl}, 0.2 \mathrm{M}$ sodium phosphate, and $20 \mathrm{mM}$ imidazole) and $10 \%$ buffer $\mathrm{B}(0.5 \mathrm{M} \mathrm{NaCl}, 0.2 \mathrm{M}$ sodium phosphate, and $0.5 \mathrm{M}$ imidazole) followed by elution with $100 \%$ buffer B. Fractions containing purified recombinant enzymes were pooled and desalted by Sephadex G50 column (GE Healthcare) size-exclusion chromatography into lysis buffer (Schenck et al., 2017). The purity of purified proteins were analyzed by SDS-PAGE using ImageJ software (Schneider et al., 2012). All protein purification steps were performed at $4^{\circ} \mathrm{C}$ unless stated otherwise.

\section{ADH and PDH Assays}

$\mathrm{ADH}$ and $\mathrm{PDH}$ assays were performed using purified recombinant enzymes for SsTyrA Wild-type (Wt) and D208N mutant, and MhTyrA Wt and Q227E mutant, while the E. coli cell lysate was used for OiTyrA as expression and purification of this enzyme was unsuccessful. Reactions contained $0.8 \mathrm{mM}$ substrate (arogenate or prephenate) and $0.8 \mathrm{mM}$ cofactor $\left(\mathrm{NADP}^{+}\right.$or $\mathrm{NAD}^{+}$) together with reaction buffer [ $25 \mathrm{mM}$ HEPES $\mathrm{pH}$ 7.6, $50 \mathrm{mM} \mathrm{KCl}, 10 \%$ (v/v) ethylene glycol]. For OiTyrA assays containing cell lysates, reactions were incubated for $45 \mathrm{~min}$ and analyzed using HPLC as previously reported (Schenck et al., 2015). For pure enzymes, reactions were monitored every $10-15 \mathrm{~s}$ for reduced cofactor at $\mathrm{A}_{340 \mathrm{~nm}}$ using a microplate reader (Tecan Genios) in a reaction volume of $30 \mu \mathrm{L}$. Kinetic parameters of purified recombinant enzymes were determined from assays containing varying concentrations of arogenate (39.1 $\mu \mathrm{M}-5 \mathrm{mM})$ or prephenate $(46.9 \mu \mathrm{M}-6 \mathrm{mM})$ substrate, $0.8 \mathrm{mM}$ of the preferred cofactor and monitored every $10-15 \mathrm{~s}$ for production of reduced cofactor at $A_{340 \mathrm{~nm}}$ using a microplate reader (Tecan Genios). Kinetic parameters were determined by fitting initial velocity data to the Michaelis-Menten equation using Origin software (OriginLab) from technical replicate assays $(n=3)$. Arogenate substrate was prepared by enzymatic conversion of prephenate (Sigma-Aldrich) as previously reported (Maeda et al., 2010). Enzyme assays were quantified using the $\mathrm{A}_{340 \mathrm{~nm}}$ of a standard curve of reduced cofactor (NADPH or $\mathrm{NADH}$ ) and activity is expressed as Kat/mg (moles of product produced per second per mg protein). All enzyme assays were conducted at a reaction time and protein concentration that were in the linear range and proportional to reaction velocity.

\section{Modeling Microbial TyrA Enzymes}

Homology models were made using SWISS-MODEL (Biasini et al., 2014) with default parameters to predict the structures of divergent TyrA enzymes. Enzymes that are more closelyrelated to plants (e.g., SsTyrA and MhTyrA) were modeled using the GmPDH1 structure as the template, though this resulted in a poor model for BdTyrA, which falls within the outgroup. BdTyrA was additionally modeled using Synechocystis sp. PCC6803 ADH. Homology models were visualized using PyMOL.

\section{RESULTS}

\section{Phylogenetic Relationship of Plant and Microbial TyrAs}

Previous studies suggested that plant TyrAs are not derived from an eukaryotic ancestor or through cyanobacterial endosymbiosis 


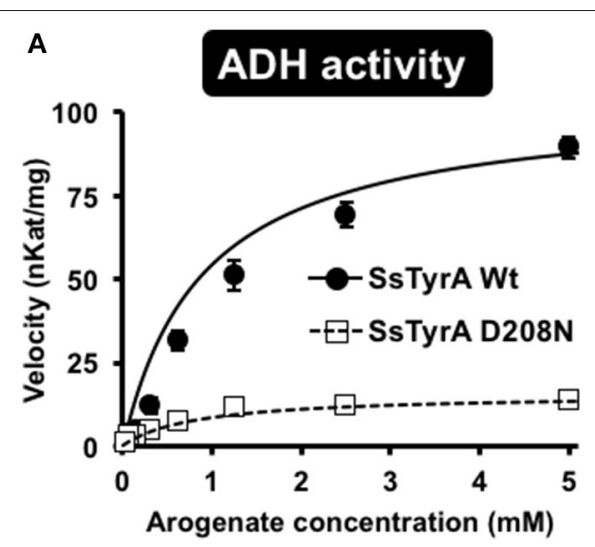

C

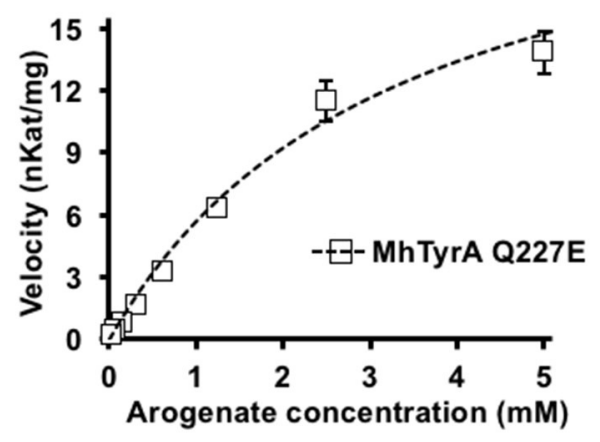

B

PDH activity

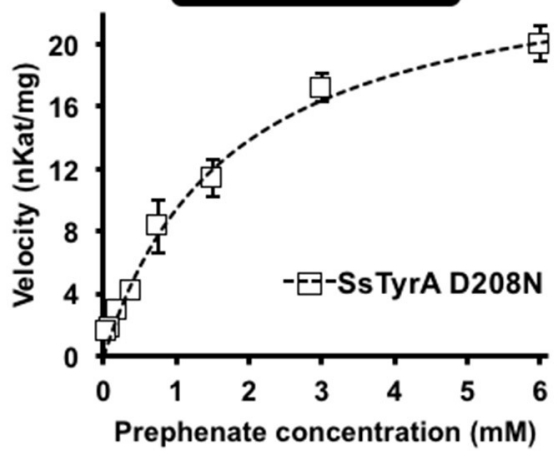

D

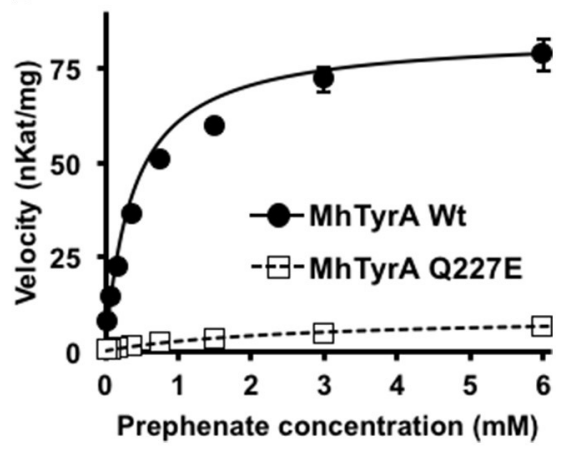

FIGURE 4 | Kinetic analysis of Wt and mutant enzymes of SsTyrAa and MhTyrAp. Kinetic analyses were performed with SsTyrAa Wt (filled circle) and D208N mutant (open square) enzymes with arogenate (A) and SsTyr $\mathrm{A}_{a}$ D208N mutant (open square) with prephenate (B). Kinetic analyses were also performed with MhTyrAp Wt (filled circle) and Q227E mutant (open square) enzymes using arogenate (C) and prephenate (D). Initial velocity values at each substrate concentration were fit to the Michaelis-Menten equation using Origin software. Kinetic analyses were conducted for MhTyrAp Wt using $3.41 \mu \mathrm{g}$ of purified recombinant enzyme, and 4.56 and 2.28 $\mu \mathrm{g}$ of purified recombinant Q227E using prephenate and arogenate, respectively. Kinetic analyses conducted for SsTyrAa Wt used 0.30 $\mu \mathrm{g}$ of purified recombinant enzyme, and $0.39 \mu \mathrm{g}$ of purified recombinant D208N. Data are means \pm SEM ( $n=3$ independent experiments). Error bars smaller than symbols are not shown.

because they are most similar to other microbes including some proteobacteria (Bonner et al., 2008; Reyes-Prieto and Moustafa, 2012; Dornfeld et al., 2014; Schenck et al., 2017); however, their precise origin was unclear. To resolve the phylogenetic relationship of TyrA orthologs from divergent organisms including plants and microbes, here we performed structureguided phylogenetic analyses using PROMALS3D to achieve alignment of TyrA orthologs with low sequence similarities (see section Materials and Methods; Pei and Grishin, 2007). Three distinct clades were identified that contain: plant TyrAs together with those from algae, spirochaetes, $\alpha$ - and $\delta$-proteobacteria (clade I, shaded blue in Figure 2, Figure S1), TyrA orthologs from some archaea, fungi, $\gamma$-proteobacteria, and chloroflexi (clade II, shaded green), and TyrA orthologs from various microbes, which formed the outgroup and contains previously characterized microbial TyrA orthologs from Synechocystis sp. PCC 6803 and Aquifex aeolicus having very low sequence similarity ( $\sim 30 \%)$ to plant TyrAs (clade III, Figure 2, Figure S1). Interestingly, TyrAs from some spirochaetes lineages (some of which are known to cause harmful human diseases like Lyme disease; Pritt et al., 2016) formed a subclade with plant and algae TyrAs within clade I using various phylogenetic methods (Figure 2, Figure S1). These data suggest that Plantae TyrA may have been acquired through horizontal gene transfer (HGT) from an ancestor of one of these closely-related microbes.

\section{Microbial TyrA Orthologs Containing an Acidic 222 Residue Prefer ADH Over PDH Activity}

The amino acid sequence alignment of TyrAs showed that the Asp222 residue, which is conserved across plant TyrA $\mathrm{A}_{\mathrm{a}}$ (Schenck et al., 2017) was also highly conserved in clade I (Figure 2). On the other hand, most sequences in clade II, including some archaea TyrA, have a non-acidic Gln residue at the corresponding 222 position (Figure 2), similar to legume $\mathrm{TyrA}_{\mathrm{p}}$ enzymes (Schenck et al., 2017). Homology models of representative TyrA from clade I-Arabidopsis thaliana ADH (AtADH2, Plantea; Rippert and Matringe, 2002) and S. smaragdinae DSM 11293 (SsTyrA, spirochaetes) - and clade II-M. harundinacea (MhTyrA, archaea) - generated using GmPDH1 structure as the template indeed showed that their acidic and non-acidic residues, respectively, correspond to Asp222 in the active site of plant TyrA (Figure S2). These data together suggest that TyrAs from clade I are likely arogenate-specific $\operatorname{TyrA}_{a}$ enzymes, whereas more distantly-related microbial TyrAs from clade II are likely prephenate-specific TyrA $A_{p}$ enzymes. 
TABLE 1 | Kinetic analysis of MhTyrAp and SsTyrAa Wt and mutant enzymes.

\begin{tabular}{|c|c|c|c|c|}
\hline Enzyme & Substrate & $k_{\text {cat }}\left(\mathrm{s}^{-1}\right)$ & $\boldsymbol{K}_{\mathrm{m}}(\mathrm{mM})$ & $\begin{array}{l}k_{\text {cat }} / K_{\mathrm{m}} \\
\left(\mathrm{mM}^{-1} \mathrm{~s}^{-1}\right)\end{array}$ \\
\hline SsTyrAa Wt & Arogenate & $3.44 \pm 0.31$ & $0.901 \pm 0.15$ & $4.03 \pm 1.01$ \\
\hline SsTyrAa Wt & Prephenate & N.D. & N.D. & N.D. \\
\hline SsTyrAa D208N & $A$ & 0.45 & 16 & 14 \\
\hline SsTyrAa D208N & Prephenate & $0.863 \pm 0.12$ & $1.742 \pm 0.63$ & $0.587 \pm 0.40$ \\
\hline MhTyrAp Wt & Arogenate & N.D. & N.D. & N.D. \\
\hline MhTyrAp Wt & Prephenate & $2.44 \pm 0.38$ & $0.378 \pm 0.02$ & $6.44 \pm 0.54$ \\
\hline MhTyrAp Q227E & Arogenate & $0.704 \pm 0.03$ & $3.290 \pm 0.22$ & $0.213 \pm 0.05$ \\
\hline MhTyrAp Q227E & Prephenate & $0.285 \pm 0.02$ & $2.669 \pm 0.32$ & $0.107 \pm 0.04$ \\
\hline
\end{tabular}

N.D. activity below detection limit, Kinetic parameters are the average of three replicate experiments $(n=3) \pm$ SEM. Kinetic analyses were conducted as described in Figure 4 legend.

To experimentally test if TyrAs from clade I have $\mathrm{ADH}$ activity, representative TyrA orthologs from two distinct subclades of clade I, spirochaetes (SsTyrA) and $\alpha$-proteobacteria (O. intermedium; OiTyrA, Figure 2, Figure S1), were expressed in E. coli as recombinant enzymes and biochemically characterized. SsTyrA and OiTyrA were chosen as they are located at key phylogenetic boundaries within clade I and contain residues required for cofactor binding and catalysis (Figure S2, Data S2). Purified SsTyrA recombinant enzyme showed ADH activity with a slight preference for $\mathrm{NAD}^{+}$over $\mathrm{NADP}^{+}$cofactor; however, PDH activity was not detectable (Figure 3A). Similarly, the E. coli cell lysate expressing OiTyrA had ADH but not PDH activity and strongly preferred $\mathrm{NAD}^{+}$over $\mathrm{NADP}^{+}$cofactor (Figure $3 \mathbf{B}$ ), although the purification of OiTyrA was not successful due to low expression. These results demonstrate that microbial TyrA orthologs from clade I, which contain an acidic residue at the corresponding 222 position (Figure 2, Data S2), are arogenatespecific TyrA $\mathrm{a}_{\mathrm{a}}$ enzymes.

\section{An Archaeon TyrA Containing a Non-acidic Residue Prefers PDH Over ADH Activity}

To test if TyrA orthologs from clade II, which contain a nonacidic residue at the corresponding 222 position, are prephenate specific TyrA $\mathrm{p}_{\mathrm{p}}$ enzymes, a representative archaeon TyrA from M. harundinacea (MhTyrA) was biochemically characterized. MhTyrA was chosen as no TyrAs from its subclade of clade II have previously been characterized (Figure 2). Also, MhTyrA is a monofunctional enzyme, while some archaea, fungi, and $\gamma$ proteobacteria orthologs in clade II are bifunctional and have a chorismate mutase enzyme domain (Hudson et al., 1984; Shlaifer et al., 2017). MhTyrA was expressed in E. coli and the recombinant enzyme was purified to homogeneity using affinity-chromatography (Figure S3) and used for biochemical analyses. Unlike plant and microbial TyrA $\mathrm{a}_{\mathrm{a}}$ orthologs from clade I, MhTyrA showed strong $\mathrm{PDH}$ and very weak $\mathrm{ADH}$ activity (Figure 3C). Interestingly, MhTyrA strongly preferred $\mathrm{NADP}^{+}$ over $\mathrm{NAD}^{+}$cofactor (Figure 3C), like plant TyrAs (Gaines et al., 1982; Connelly and Conn, 1986). These results suggest that TyrA orthologs from clade II that have a non-acidic residue at the corresponding 222 position are $\mathrm{TyrA}_{\mathrm{p}}$ enzymes that strongly prefer prephenate over arogenate substrate.

\section{A Single D208N Mutation Introduces PDH Activity in a Spirochaetes TyrAa}

To test if the acidic residue at the corresponding 222 position in spirochaetes SsTyrA $\mathrm{A}_{\mathrm{a}}$ is involved in its substrate specificity, the $\mathrm{D} 208 \mathrm{~N}$ mutation that converts the active site acidic residue into a non-acidic residue was introduced in SsTyrA. The purified recombinant SsTyrAa D208N enzyme (Figure S3) drastically decreased its original $\mathrm{ADH}$ activity (Figure 4A), but now exhibited PDH activity (Figure 4B), with a slight alteration in cofactor specificity in the mutant compared to $\mathrm{Wt}$ (Figure S4).

Kinetic analyses showed that SsTyrA $\mathrm{a}$ Wt enzyme did not accept prephenate and had a $K_{\mathrm{m}}$ for arogenate of $901 \mu \mathrm{M}$ (Table 1), which is substantially higher than previously reported plant and microbial TyrA enzymes (Rippert and Matringe, 2002; Bonvin et al., 2006; Ku et al., 2010; Schenck et al., 2015). The SsTyrA $_{a}$ D208N mutant exhibited a seven-fold lower catalytic efficiency $\left(k_{\text {cat }} / K_{\mathrm{m}}\right)$ with arogenate than Wt, though the $K_{\mathrm{m}}$ was not altered (Table 1). SsTyrA ${ }_{a}$ D208N showed significant $\mathrm{PDH}$ activity that was absent in Wt, though its $K_{\mathrm{m}}$ was still much higher than previously reported TyrA enzymes (Rippert and Matringe, 2002; Bonvin et al., 2006; Ku et al., 2010; Schenck et al., 2015) and had poor catalytic efficiency (Table 1). These results suggest that a single mutation of the active site acidic residue to a non-acidic residue can alter the substrate specificity of spirochaetes TyrA, similar to plant TyrAs (Schenck et al., 2017).

\section{A Single Q227E Mutation Introduces ADH Activity in an Archaeon TyrA}

To test if the non-acidic residue of $\mathrm{MhTyrA}_{p}$ at the corresponding 222 position (Gln227) is involved in prephenate substrate specificity, site-directed mutagenesis was performed on $\mathrm{MhTyrA}_{\mathrm{p}}$ to replace Gln227 with acidic Glu and generate the MhTyrA $_{p}$ Q227E mutant. The purified recombinant MhTyrA Q227E enzyme (Figure S3) showed decreased PDH activity (Figure 4D) with a substantial gain of ADH activity (Figure 4C, Table 1) without altering cofactor preference (Figure S4).

Further kinetic analyses showed that Wt $\mathrm{MhTyrA}_{\mathrm{p}}$ had a $K_{\mathrm{m}}$ of $378 \mu \mathrm{M}$ and turnover rate $\left(k_{\text {cat }}\right)$ of $2.4 \mathrm{~s}^{-1}$ using prephenate substrate and $\mathrm{NADP}^{+}$cofactor (Figure 4, Table 1), which are comparable to previously characterized microbial TyrA $_{\mathrm{p}}$ enzymes (Bonvin et al., 2006; Ku et al., 2010). The very

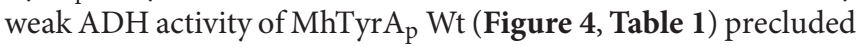
it from kinetic analysis using arogenate.

The Q227E mutant, on the other hand, exhibited almost 10 -fold reduction in $K_{\mathrm{m}}$ for prephenate $(2.7 \mathrm{mM})$, while the catalytic efficiency $\left(k_{\text {cat }} / K_{\mathrm{m}}\right)$ was reduced by 60 -fold ( 0.1 vs. 6.4 $\mathrm{mM}^{-1} \mathrm{~s}^{-1}$, Figure 4D, Table 1). The Q227E mutant displayed substantial ADH activity compared to the Wt enzyme with a $K_{\mathrm{m}}$ for arogenate of $3.3 \mathrm{mM}$, similar to that of Q227E for prephenate (2.7 mM, Figure 4C, Table 1) though still 10-fold higher than that of the Wt enzyme for prephenate (Figure 4D, Table 1) and 
other previously characterized TyrA $\mathrm{a}_{\mathrm{a}}$ enzymes (Bonvin et al., 2006; Ku et al., 2010; Schenck et al., 2015, 2017). The Q227E mutant had roughly two-fold higher catalytic efficiency with arogenate than with prephenate (0.2 vs. $0.1 \mathrm{mM}^{-1} \mathrm{~s}^{-1}$, Figure 3). These results demonstrate that the Q227E mutation can shift the substrate preference of $\mathrm{MhTyrA}_{\mathrm{p}}$ from prephenate to arogenate, suggesting that the single residue is responsible for substrate specificity of archaea TyrA $\mathrm{A}_{\mathrm{p}}$ enzymes.

\section{DISCUSSION}

Previous studies suggest that microbes predominantly use a PDH-mediated pathway to synthesize Tyr, whereas plants mainly use an ADH-mediated Tyr pathway (Jensen and Pierson, 1975; Bentley, 1990; Siehl, 1999; Rippert and Matringe, 2002; Maeda and Dudareva, 2012; Schenck et al., 2015, 2017). In this study, structure-guided phylogenetic analyses from diverse organisms identified $\mathrm{ADH}$-like sequences in some bacteria, e.g., spirochaetes, $\alpha$ - and $\delta$-proteobacteria, which form a monophyletic clade with plant TyrAs (Figure 2, Figure S1). Biochemical characterization further demonstrated that TyrAs from spirochaetes and $\alpha$-proteobacteria indeed have $\mathrm{ADH}$, but not PDH activity (Figures 3A,B). A native TyrA enzyme purified from the $\alpha$-proteobacteria $P$. immobile, which belongs to the same $\alpha$-proteobacteria genus found in clade I, was also previously shown to have $\mathrm{ADH}$, but not PDH activity (Mayer et al., 1985). Therefore, our study revealed that arogenate-specific TyrA enzymes are more widely distributed in microbes than previously thought.

Previous evolutionary studies revealed that plant aromatic amino acid pathway enzymes are derived from a wide range of, and sometimes unexpected microbial origins (Richards et al., 2006; Reyes-Prieto and Moustafa, 2012; Dornfeld et al., 2014). For example, plant shikimate kinase is most likely derived from cyanobacteria endosymbiosis (Richards et al., 2006) whereas plant prephenate aminotransferase and arogenate dehydratase involved in Phe biosynthesis are sister to Chlorobi/Bacteroidetes orthologs (Dornfeld et al., 2014). However, the evolutionary origin of plant TyrAs is currently unknown. TyrAs from some spirochaetes were more closely-related to plant and algae $\operatorname{Tyr}_{a} \mathrm{~s}$ than other microbial TyrAs from clade I (Figure 2, Figure S1) and, like Plantae TyrA $\mathrm{a}_{\mathrm{a}}$ enzymes, had a conserved acidic residue at the corresponding 222 position. BlastP searches across different spirochaetes genomes showed that plant-like TyrAs are restricted to the order Spirochaetales, and absent in Leptospirales, Brevinematales, and Brachyspirales (Figure S5; Gupta et al., 2013). Thus, the current result suggests that the common ancestor of algae and plants acquired a $\mathrm{TyrA}_{\mathrm{a}}$ enzyme from a spirochaetes ancestor likely through a novel HGT event, rather than from an $\alpha$-proteobacteria through mitochondria symbiosis (Gray et al., 1999).

The archaeon MhTyrA from clade II preferred PDH over ADH activity (Figure 3C) and had a non-acidic residue at the 222 position (Figure 2, Figure S1). This is consistent with previouslycharacterized clade II TyrA enzymes from $\gamma$-proteobacteria and fungi, which also preferred $\mathrm{PDH}$ over $\mathrm{ADH}$ activity (Mannhaupt et al., 1989; Christendat and Turnbull, 1999; Chiu et al., 2010) though they belonged to distinct subclades (Figure 2). As almost all TyrA sequences within clade II have a nonacidic residue (Gln or Asn) at the corresponding 222 position, except for Chloroflexi TyrAs (Figure 2, Figure S1), they are likely prephenate-specific TyrA $\mathrm{T}_{\mathrm{p}}$ enzymes. Previously characterized microbial TyrA $\mathrm{p}_{\mathrm{p}}$ enzymes had similar $K_{\mathrm{m}}$ with prephenate as MhTyrA $_{p}$ (Figure 4, Table 1); however the catalytic efficiency of MhTyrA $_{p}$ was lower than previously characterized TyrA enzymes (Bonvin et al., 2006; Ku et al., 2010). This implies that MhTyrA may also have alternative in vivo substrates and further genetic studies are needed to determine the in vivo function of MhThrA $_{\mathrm{p}}$.

In plant TyrAs, an acidic residue at the corresponding 222 position confers $\mathrm{ADH}$ activity by directly interacting with the side chain amine of arogenate, and when mutated to a non-acidic Asn, switches to PDH activity (Schenck et al., 2017). Consistently, in OiTyrA $\mathrm{a}_{\mathrm{a}}$ and $\mathrm{SsTyrA}_{\mathrm{a}}$, which contain acidic residues at the corresponding 222 position prefer $\mathrm{ADH}$ activity (Figure 3). Furthermore, mutation of the corresponding acidic residue into a non-acidic residue on $\mathrm{SsTyr}_{\mathrm{a}}$ introduced novel PDH activity (Figure 4, Table 1). The reciprocal mutation (Gln to Glu) on $\mathrm{MhTyrA}_{\mathrm{p}}$ reduced $\mathrm{PDH}$ activity while gaining $\mathrm{ADH}$ activity (Figure 4, Table 1), further supporting that the corresponding 222 position in microbial TyrA enzymes is also important for their substrate specificity. However, ADH activity of $\mathrm{MhTyrA}_{\mathrm{p}}$ Q227E was only two-fold higher than its PDH activity and still 30-fold lower than PDH of $\mathrm{MhTyrA}_{\mathrm{p}} \mathrm{Wt}$. Also, $\mathrm{PDH}$ activity

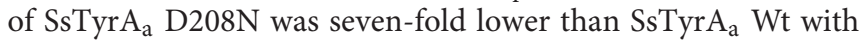
arogenate (Figure 4, Table 1). These results suggest that residues besides the corresponding 222 substrate specificity determining residue likely contribute to overall catalytic activity of microbial TyrA enzymes. These data together suggest that mutation of the non-acidic to an acidic residue at the corresponding 222 position played a key role in the evolution of arogenate-specific TyrA enzymes in microbes from clade I that gave rise to plant TyrAs.

The outgroup (clade III) appears to contain TyrA enzymes with both PDH and ADH activity (Xia and Jensen, 1990; Zhao et al., 1993; Bonvin et al., 2006; Legrand et al., 2006). Homology models of microbial TyrAs from the outgroup (e.g., Bifidobacterium dentium TyrA; BdTyrA) were compared to previously crystallized GmPDH1 (Schenck et al., 2017) and Synechocystis ADH (Legrand et al., 2006) to determine if the substrate specificity mechanism of TyrAs from clade I and II are also conserved in clade III TyrAs (Figure S6). The global conformations of these divergent TyrA proteins from clade I and III are similar in structure, though there are some differences, such as additional $\alpha$-helices around the C-terminal dimerization domain (Figure S6). All structures have conserved catalytic Ser101 and His124 (Christendat and Turnbull, 1999; Sun et al., 2006) that directly interact with ring hydroxyl of arogenate and prephenate substrate (Schenck et al., 2017), suggesting that the key catalytic residues have been maintained across divergent TyrAs. However, the two loop regions surrounding and recognizing the substrate side chain by the 222 residue (Schenck et al., 2017), are not well conserved in clade III as compared to clade I TyrAs (Figure S6). This makes it difficult to 
confidently assign a corresponding residue in clade III TyrAs to the 222 position of clade I TyrAs (Figure 2, Data S2). Thus, clade III TyrAs likely use a different molecular mechanism(s) for their substrate specificity than plant and closely-related microbial TyrAs from clade I and II.

Alteration of substrate specificity of microbial TyrA enzymes provides another example of a single active site residue that modifies substrate specificity of plant and microbial enzymes (Louie et al., 2006; He et al., 2011; Fan et al., 2016). Substitutions between active site $\mathrm{Val}$ and Phe residues switch the acylCoA substrate specificity of Solanum acylsugar acyltransferases that belong to the BAHD acyltransferase family (Fan et al., 2016). A single mutation of the conserved active site Leu residue of isopropylmalate dehydrogenases involved in leucine biosynthesis is sufficient to convert their specificity to 3( $2^{\prime}$-methylthio)ethylmalate, an intermediate of the methionine chain-elongation pathway required for aliphatic glucosinolate biosynthesis (He et al., 2011). Mutating His89 of a microbial Tyr ammonia lyase (TAL) into Phe also switches its substrate specificity to prefer Phe instead of Tyr (Louie et al., 2006). Many of these examples are the result of gene duplications followed by neofunctionalization by a single key amino acid mutation, resulting in a novel substrate specificity and recruitment to a different metabolic pathway (Leong and Last, 2017). Despite the single amino acid mutation that switches substrate specificity of TyrA dehydrogenases, they are still involved in the same Tyr biosynthesis but alters the pathway architecture.

In conclusion, the current study revealed that arogenatespecific $\mathrm{TyrA}_{\mathrm{a}}$ enzymes evolved in some bacterial lineages, through the acquisition of an acidic residue at the 222 position, which later gave rise to the TyrAs of algae and land plants likely through a novel HGT event. More recently, the same residue was mutated back to a non-acidic residue uniquely in legume plants, which resulted in prephenate-

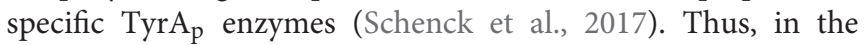
course of TyrA enzyme evolution, microbial TyrA $\mathrm{p}_{\mathrm{p}}$ were converted into microbial $\mathrm{TyrA}_{\mathrm{a}}$ and then to legume-specific

\section{REFERENCES}

Bentley, R. (1990). The shikimate pathway - a metabolic tree with many branches. Crit. Rev. Biochem. Mol. Biol. 25, 307-384. doi: 10.3109/10409239009090615

Biasini, M., Bienert, S., Waterhouse, A., Arnold, K., Studer, G., Schmidt, T., et al. (2014). SWISS-MODEL: modelling protein tertiary and quaternary structure using evolutionary information. Nucleic Acids Res. 42, W252-W258. doi: 10.1093/nar/gku340

Bonner, C. A., Disz, T., Hwang, K., Song, J., Vonstein, V., Overbeek, R., et al. (2008). Cohesion group approach for evolutionary analysis of TyrA, a protein family with wide-ranging substrate specificities. Microbiol. Mol. Biol. Rev. 72, 13-53. doi: 10.1128/MMBR.00026-07

Bonvin, J., Aponte, R. A., Marcantonio, M., Singh, S., Christendat, D., and Turnbull, J. L. (2006). Biochemical characterization of prephenate dehydrogenase from the hyperthermophilic bacterium Aquifex aeolicus. Protein Sci. 15, 1417-1432. doi:10.1110/ps.051942206

Chiu, H. J., Abdubek, P., Astakhova, T., Axelrod, H. L., Carlton, D., Clayton, T., et al. (2010). The structure of Haemophilus influenzae prephenate dehydrogenase suggests unique features of bifunctional TyrA enzymes.
$\operatorname{TyrA}_{p}$ by altering the same active site residue from a nonacidic to an acidic, and then back to a non-acidic residue. Previous studies proposed that the ubiquitous presence of the ADH-mediated Tyr pathway among photosynthetic organisms is to avoid futile cycling of tocopherol and plastoquinone biosynthesis from HPP (Siehl, 1999; Graindorge et al., 2014). Identification of arogenate-specific TyrA among many nonphotosynthetic microbes may require revisiting the biological significance of the $\mathrm{ADH}$ vs. PDH-mediated Tyr biosynthetic pathways in diverse organisms. Given that arogenate and prephenate substrate specificity of TyrAs can be readily converted by a single residue (Figure 4, Table 1; Schenck et al., 2017), there must be significant selection pressure to maintain the acidic 222 residue and thus $\mathrm{ADH}$ activity in many organisms. The molecular mechanism and the key amino acid residue regulating the biochemical properties of diverse TyrAs also enables the optimization of Tyr biosynthesis via two alternative Tyr biosynthetic pathways in both plants and microbes, for enhanced production of pharmaceutically important natural products derived from Tyr (e.g., morphine and vitamin $\mathrm{E})$.

\section{AUTHOR CONTRIBUTIONS}

CAS and YM performed experiments and analyzed data; CAS and HAM conceived the experiments, and CAS wrote the manuscript. All authors read and edited the manuscript.

\section{ACKNOWLEDGMENTS}

This work was supported by the National Science Foundation (IOS-1354971 to HAM).

\section{SUPPLEMENTARY MATERIAL}

The Supplementary Material for this article can be found online at: https://www.frontiersin.org/articles/10.3389/fmolb. 2017.00073/full\#supplementary-material
Acta Crystallogr. Sect. F Struct. Biol. Cryst. Commun. 66, 1317-1325. doi: $10.1107 /$ S1744309110021688

Christendat, D., and Turnbull, J. L. (1999). Identifying groups involved in the binding of prephenate to prephenate dehydrogenase from Escherichia coli. Biochemistry 38, 4782-4793. doi: 10.1021/bi982673o

Connelly, J. A., and Conn, E. E. (1986). Tyrosine biosynthesis in Sorghum bicolor: isolation and regulatory properties of arogenate dehydrogenase. $Z$. Naturforschung C 41, 69-78.

Dornfeld, C., Weisberg, A. J., K., C, R., Dudareva, N., Jelesko, J. G., and Maeda, H. A. (2014). Phylobiochemical characterization of class-Ib aspartate/prephenate aminotransferases reveals evolution of the plant arogenate phenylalanine pathway. Plant Cell 26, 3101-3114. doi: 10.1105/tpc.114.127407

Falk, J., and Munné-Bosch, S. (2010). Tocochromanol functions in plants: antioxidation and beyond. J. Exp. Bot. 61, 1549-1566. doi: 10.1093/jxb/ erq030

Fan, P., Miller, A. M., Schilmiller, A. L., Liu, X., Ofner, I., Jones, A. D., et al. (2016). In vitro reconstruction and analysis of evolutionary variation of the tomato acylsucrose metabolic network. Proc. Natl. Acad. Sci. U.S.A. 113, E239-E248. doi: $10.1073 /$ pnas. 1517930113 
Fazel, A. M., Bowen, J. R., and Jensen, R. A. (1980). Arogenate (pretyrosine) is an obligatory intermediate of L-tyrosine biosynthesis: confirmation in a microbial mutant. Proc. Natl. Acad. Sci. U.S.A. 77, 1270-1273. doi: 10.1073/pnas.77.3.1270

Fernstrom, J. D., and Fernstrom, M. H. (2007). Tyrosine, phenylalanine, and catecholamine synthesis and function in the brain. J. Nutr. 137, 1539S-1547S. Available online at: http://jn.nutrition.org/content/137/6/1539S.long

Fitzpatrick, P. F. (1999). Tetrahydropterin-dependent amino acid hydroxylases. Annu. Rev. Biochem. 68, 355-381. doi: 10.1146/annurev.biochem.68.1.355

Gaines, C. G., Byng, G. S., Whitaker, R. J., and Jensen, R. A. (1982). L-Tyrosine regulation and biosynthesis via arogenate dehydrogenase in suspensioncultured cells of Nicotiana silvestris Speg. et Comes. Planta 156, 233-240. doi: 10.1007/BF00393730

Gamborg, O. L., and Keeley, F. W. (1966). Aromatic metabolism in plants I. A study of the prephenate dehydrogenase from bean plants. Biochim. Biophys. Acta 115, 65-72. doi: 10.1016/0304-4165(66)90049-3

Gandía-Herrero, F., and García-Carmona, F. (2013). Biosynthesis of betalains: yellow and violet plant pigments. Trends Plant Sci. 18, 334-343. doi: 10.1016/j.tplants.2013.01.003

Gleadow, R. M., and Møller, B. L. (2014). Cyanogenic glycosides: synthesis, physiology, and phenotypic plasticity. Annu. Rev. Plant Biol. 65, 155-185. doi: 10.1146/annurev-arplant-050213-040027

Graindorge, M., Giustini, C., Kraut, A., Moyet, L., Curien, G., and Matringe, M. (2014). Three different classes of aminotransferases evolved prephenate aminotransferase functionality in arogenate-competent microorganisms. J. Biol. Chem. 289, 3198-3208. doi: 10.1074/jbc.M113.486480

Gray, M. W., Burger, G., and Lang, B. F. (1999). Mitochondrial evolution. Science 283, 1476-1481. doi: 10.1126/science.283.5407.1476

Gupta, R. S., Mahmood, S., and Adeolu, M. (2013). A phylogenomic and molecular signature based approach for characterization of the phylum Spirochaetes and its major clades: proposal for a taxonomic revision of the phylum. Front. Microbiol. 4:322. doi: 10.3389/fmicb.2013.00322

He, Y., Galant, A., Pang, Q., Strul, J. M., Balogun, S. F., Jez, J. M., et al. (2011). Structural and functional evolution of isopropylmalate dehydrogenases in the leucine and glucosinolate pathways of Arabidopsis thaliana. J. Biol. Chem. 286, 28794-28801. doi: 10.1074/jbc.M111.262519

Hudson, G. S., Wong, V., and Davidson, B. E. (1984). Chorismate mutase/prephenate dehydrogenase from Escherichia coli K12: purification, characterization, and identification of a reactive cysteine. Biochemistry 23, 6240-6249. doi: 10.1021/bi00320a054

Jensen, R. A., and Pierson, D. L. (1975). Evolutionary implications of different types of microbial enzymology for L-tyrosine biosynthesis. Nature 254, 667-671. doi: $10.1038 / 254667 \mathrm{a} 0$

Jones, D. T., Taylor, W. R., and Thornton, J. M. (1992). The rapid generation of mutation data matrices from protein sequences. Comput. Appl. Biosci. 8, 275-282. doi: 10.1093/bioinformatics/8.3.275

Ku, H., Park, S., Yang, I., and Kim, S. (2010). Expression and functional characterization of prephenate dehydrogenase from Streptococcus mutans. Process Biochem. 45, 607-612. doi: 10.1016/j.procbio.2009.12.006

Kumar, S., Stecher, G., and Tamura, K. (2016). MEGA7: molecular evolutionary genetics analysis version 7.0 for bigger datasets. Mol. Biol. Evol. 33, 1870-1874. doi: $10.1093 / \mathrm{molbev} / \mathrm{msw} 054$

Legrand, P., Dumas, R., Seux, M., Rippert, P., Ravelli, R., Ferrer, J. L., et al. (2006). Biochemical characterization and crystal structure of Synechocystis arogenate dehydrogenase provide insights into catalytic reaction. Structure 14, 767-776. doi: 10.1016/j.str.2006.01.006

Leong, B. J., and Last, R. L. (2017). Promiscuity, impersonation and accommodation: evolution of plant specialized metabolism. Curr. Opin. Struct. Biol. 47, 105-112. doi: 10.1016/j.sbi.2017.07.005

Louie, G. V., Bowman, M. E., Moffitt, M. C., Baiga, T. J., Moore, B. S., and Noel, J. P. (2006). Structural determinants and modulation of substrate specificity in phenylalanine-tyrosine ammonia-lyases. Chem. Biol. 13, 1327-1338. doi: 10.1016/j.chembiol.2006.11.011

Maeda, H., and Dudareva, N. (2012). The shikimate pathway and aromatic amino acid biosynthesis in plants. Annu. Rev. Plant Biol. 63, 73-105. doi: 10.1146/annurev-arplant-042811-105439

Maeda, H., Shasany, A. K., Schnepp, J., Orlova, I., Taguchi, G., Cooper, B. R., et al. (2010). RNAi suppression of Arogenate Dehydratase1 reveals that phenylalanine is synthesized predominantly via the arogenate pathway in petunia petals. Plant Cell 22, 832-849. doi: 10.1105/tpc.109.073247

Mannhaupt, G., Stucka, R., Pilz, U., Schwarzlose, C., and Feldmann, H. (1989). Characterization of the prephenate dehydrogenase-encoding gene, TYR1, from Saccharomyces cerevisae. Gene 85, 303-311. doi: 10.1016/0378-1119(89)90422-8

Mayer, E., Waldner-Sander, S., Keller, B., Keller, E., and Lingens, F. (1985). Purification of arogenate dehydrogenase from Phenylobacterium immobile. FEBS Lett. 179, 208-212. doi: 10.1016/0014-5793(85)80519-6

Millner, P. A., and Barber, J. (1984). Plastoquinone as a mobile redox carrier in the photosynthetic membrane. FEBS Lett. 169, 1-6. doi: 10.1016/0014-5793(84)80277-X

Pei, J., and Grishin, N. V. (2007). PROMALS: towards accurate multiple sequence alignments of distantly related proteins. Bioinformatics 23, 802-808. doi: 10.1093/bioinformatics/btm017

Petersen, M. (2013). Rosmarinic acid: new aspects. Phytochem. Rev. 12, 207-227. doi: 10.1007/s11101-013-9282-8

Pritt, B. S., Mead, P. S., Johnson, D. K. H., Neitzel, D. F., Respicio-Kingry, L. B., Davis, J. P., et al. (2016). Identification of a novel pathogenic Borrelia species causing Lyme borreliosis with unusually high spirochaetaemia: a descriptive study. Lancet Infect. Dis. 16, 556-564. doi: 10.1016/S1473-3099(15) 00464-8

Reyes-Prieto, A., and Moustafa, A. (2012). Plastid-localized amino acid biosynthetic pathways of Plantae are predominantly composed of non-cyanobacterial enzymes. Sci. Rep. 2, 955-967. doi: 10.1038/srep 00955

Richards, T. A., Dacks, J. B., Campbell, S. A., Blanchard, J. L., Foster, P. G., McLeod, R., et al. (2006). Evolutionary origins of the eukaryotic shikimate pathway: gene fusions, horizontal gene transfer, and endosymbiotic replacements. Eukaryot. Cell 5, 1517-1531. doi: 10.1128/EC.00106-06

Rippert, P., and Matringe, M. (2002). Purification and kinetic analysis of the two recombinant arogenate dehydrogenase isoforms of Arabidopsis thaliana. Eur. J. Biochem. 269, 4753-4761. doi: 10.1046/j.1432-1033.2002.03172.x

Rubin, J. L., and Jensen, R. A. (1979). Enzymology of L-tyrosine biosynthesis in mung bean (Vigna radiata [L.] Wilczek). Plant Physiol. 64, 727-734. doi: $10.1104 /$ pp.64.5.727

Saitou, N., and Nei, M. (1987). The neighbor-joining method: a new method for reconstructing phylogenetic trees. Mol. Biol. Evol. 4, 406-425.

Sato, F., Inui, T., and Takemura, T. (2007). Metabolic engineering in isoquinoline alkaloid biosynthesis. Curr. Pharm. Biotechnol. 8, 211-218. doi: 10.2174/138920107781387438

Schenck, C. A., Chen, S., Siehl, D. L., and Maeda, H. A. (2015). Non-plastidic, tyrosine-insensitive prephenate dehydrogenases from legumes. Nat. Chem. Biol. 11, 52-57. doi: 10.1038/nchembio.1693

Schenck, C. A., Holland, C. K., Schneider, M. R., Men, Y., Lee, S. G., Jez, J. M., et al. (2017). Molecular basis of the evolution of alternative tyrosine biosynthetic routes in plants. Nat. Chem. Biol. 13, 1029-1035. doi: 10.1038/nchembio. 2414

Schneider, C. A., Rasband, W. S., and Eliceiri, K. W. (2012). NIH Image to ImageJ: 25 years of image analysis. Nat. Methods 9, 671-675. doi: 10.1038/ nmeth.2089

Shlaifer, I., Quashie, P. K., Kim, H. Y., and Turnbull, J. L. (2017). Biochemical characterization of TyrA enzymes from Ignicoccus hospitalis and Haemophilus influenzae: a comparative study of the bifunctional and monofunctional dehydrogenase forms. Biochim. Biophys. Acta 1865, 312-320. doi: 10.1016/j.bbapap.2016.12.014

Siehl, D. (1999). "The biosynthesis of tryptophan, tyrosine, and phenylalanine from chorismate," in Plant Amino Acids: Biochemistry and Biotechnology, ed B. Singh (New York, NY: CRC Press), 171-204.

Slominski, A., Tobin, D. J., Shibahara, S., and Wortsman, J. (2004). Melanin pigmentation in mammalian skin and its hormonal regulation. Physiol. Rev. 84, 1155-1228. doi: 10.1152/physrev.00044.2003

Subramaniam, P., Bhatnagar, R., Hooper, A., and Jensen, R. A. (1994). The dynamic progression of evolved character states for aromatic amino acid biosynthesis in gram-negative bacteria. Microbiology 140, 3431-3440. doi: 10.1099/13500872-140-12-3431

Sun, W., Singh, S., Zhang, R., Turnbull, J. L., and Christendat, D. (2006). Crystal structure of prephenate dehydrogenase from Aquifex aeolicus: 
insights into the catalytic mechanism. J. Biol. Chem. 281, 12919-12928. doi: $10.1074 /$ jbc.M511986200

Xia, T. H., and Jensen, R. A. (1990). A single clycohexadienyl dehydrogenase specifies the prephenate dehydrogenase and arogenate dehydrogenase components of the dual pathways to L-tyrosine in Pseudomonas aeruginosa. J. Biol. Chem. 265, 20033-20036.

Zhao, G., Xia, T., Ingram, L. O., and Jensen, R. A. (1993). An allosterically insensitive class of cyclohexadienyl dehydrogenase from Zymomonas mobilis. Eur. J. Biochem. 212, 157-165. doi: 10.1111/j.1432-1033.1993. tb17646.x
Conflict of Interest Statement: The authors declare that the research was conducted in the absence of any commercial or financial relationships that could be construed as a potential conflict of interest.

Copyright (c) 2017 Schenck, Men and Maeda. This is an open-access article distributed under the terms of the Creative Commons Attribution License (CC BY). The use, distribution or reproduction in other forums is permitted, provided the original author(s) or licensor are credited and that the original publication in this journal is cited, in accordance with accepted academic practice. No use, distribution or reproduction is permitted which does not comply with these terms. 